\title{
Harvesting low-grade heat energy using thermo-osmotic vapour transport through nanoporous membranes
}

\author{
Anthony P. Straub ${ }^{1}$, Ngai Yin Yip ${ }^{2}$, Shihong Lin ${ }^{3}$, Jongho Lee ${ }^{1}$ and Menachem Elimelech ${ }^{1 \star}$
}

\begin{abstract}
Low-grade heat from sources below $100^{\circ} \mathrm{C}$ offers a vast quantity of energy. The ability to extract this energy, however, is limited with existing technologies as they are not well-suited to harvest energy from sources with variable heat output or with a small temperature difference between the source and the environment. Here, we present a process for extracting energy from low-grade heat sources utilizing hydrophobic, nanoporous membranes that trap air within their pores when submerged in a liquid. By driving a thermo-osmotic vapour flux across the membrane from a hot reservoir to a pressurized cold reservoir, heat energy can be converted to mechanical work. We demonstrate operation of air-trapping membranes under hydraulic pressures up to 13 bar, show that power densities as high as $3.53 \pm 0.29 \mathrm{~W} \mathrm{~m}^{-2}$ are achievable with a $60^{\circ} \mathrm{C}$ heat source and a $20^{\circ} \mathrm{C}$ heat sink, and estimate the efficiency of a full-scale system. The results demonstrate a promising process to harvest energy from low-temperature differences $\left(<40^{\circ} \mathrm{C}\right)$ and fluctuating heat sources.
\end{abstract}

A vast amount of untapped energy exists in the form of low-grade heat from sources below $100^{\circ} \mathrm{C}$ (refs 1,2). Waste heat is one abundant example, with residual heat losses from conventional power plants and the manufacturing industry amounting to more than 8,000 TWh per year in the United States alone $e^{3,4}$. Geothermal wells and solar collectors can also supply large quantities of heat energy, and lower temperature ranges of either of these sources are more widely available and less difficult to obtain than their high-quality counterparts ${ }^{5-7}$. Despite the abundance of low-grade heat, the ability to extract energy from potential sources is limited with existing technologies due to both the small temperature difference available and the temporal variability in heat output from sources such as waste heat or solar thermal. Existing binary cycle systems utilizing organic working fluids typically require heat sources with temperatures greater than $100^{\circ} \mathrm{C}$ and are unable to tolerate fluctuations in the heat source temperature ${ }^{8-10}$. Solid-state thermoelectric devices have been evaluated for use in low-temperature ranges, but material limitations have resulted in poor efficiencies and low cost effectiveness ${ }^{11,12}$. Various thermoelectrochemical systems have also been developed for low-grade heat power generation utilizing metal complexation reactions ${ }^{13-15}$ or the temperature dependence of electrochemical redox potentials ${ }^{16-18}$ to generate power. These processes have, however, been introduced only as small-scale prototypes, and their efficiency is typically limited to less than $2 \%$ of the Carnot efficiency.

Hydrophobic, porous membranes have emerged as promising materials for a variety of applications ${ }^{19-22}$. When placed between two liquids, these membranes trap air within their pores, creating an extremely thin gas-phase barrier. A partial vapour pressure difference across the membrane arising from a temperature, concentration or hydraulic pressure gradient can be used to drive gas-phase transport of various constituents between the two liquids. This phenomenon is normally exploited for separation processes, where a thermo-osmotic (that is, temperature-driven) vapour flux is used to distil a mixture containing undesirable non-volatile constituents ${ }^{23,24}$.

We conceive an alternate thermo-osmotic energy conversion (TOEC) process in which the hydrophobic membranes utilize temperature gradients to drive a vapour flux against a hydraulic pressure difference. The resulting pressurized flow can be used to drive a turbine and generate power with a wide range of heat source temperatures, effectively converting low-temperature heat energy to useful work. We experimentally demonstrate the proposed TOEC process, showing that hydrophobic membranes are able to maintain a thin air gap between two liquid water reservoirs, even when substantial hydraulic pressure differences are applied. Low-temperature differences $\left(<40^{\circ} \mathrm{C}\right)$ are then used to generate a pressurized flow of water, and the power output of the system is determined from these thermo-osmotic flow measurements. The theoretical efficiency of a full-scale system with heat recovery is also evaluated. Overall, the proposed system enables access to vast supplies of untapped low-grade heat, demonstrating the promise of using vapour-gap membranes for pressurized applications.

\section{Working principle of thermo-osmotic energy conversion}

To extract low-temperature thermal energy, the TOEC system uses a hydrophobic, nanoporous membrane to create a thin air gap between hot and cold liquid streams (Fig. 1a). Mass transfer across this vapour-gap membrane occurs in the gas phase as liquid evaporates from one meniscus, transports across the membrane, and condenses on the opposing meniscus. The rate of vapour transport across the membrane is dependent on the partial vapour pressure difference between the menisci at either side of the membrane. The higher equilibrium vapour pressure of the hot stream therefore drives a net vapour flux across the membrane into the cold stream. The volume increase in the cold stream is partially

\footnotetext{
${ }^{1}$ Department of Chemical and Environmental Engineering, Yale University, New Haven, Connecticut 06520, USA. ${ }^{2}$ Department of Earth and Environmental Engineering, Columbia University, New York, New York 10027, USA. ${ }^{3}$ Department of Civil and Environmental Engineering, Vanderbilt University, Nashville, Tennessee 37235, USA. *e-mail: menachem.elimelech@yale.edu
} 


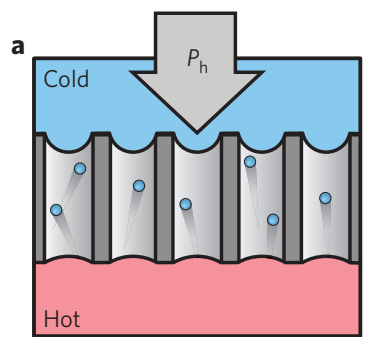

b

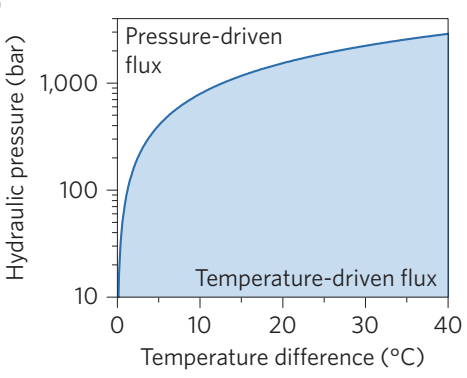

Figure 1 | Working principle of the thermo-osmotic energy conversion system. a, Schematic diagram of water vapour transport across a hydrophobic porous membrane from a hot stream to a cold stream against hydraulic pressure, $P_{h}$. b. Hydraulic pressure that can be theoretically generated with a given temperature difference across a vapour-gap membrane. Regions of operation that result in temperature-driven flux and pressure-driven flux are shown. The cold temperature is fixed at $20^{\circ} \mathrm{C}$.

restricted, generating a hydraulic pressure. Continued thermoosmotic vapour transport across the membrane into the cold stream, which becomes pressurized upon condensation, is then directed through a hydro turbine to generate electricity. In principle, many liquids can be used in this system; optimal working fluids will have a high surface tension to prevent wetting of the membrane pores, be highly affordable, and be non-hazardous to the environment. Here, we utilize water as a working fluid since it has a high surface tension, is widely available at a low cost, and is environmentally benign.

Thermo-osmotic power generation can also be achieved using other types of membranes that enable temperature-driven fluid transport and create a pressure discontinuity. For example, dense polymeric films may be suitable for the process. However, the thermal conductivity of polymeric membranes is approximately an order of magnitude greater than that of air or water vapour, leading to severe thermal losses during temperature-driven operation, and thermo-osmotic fluxes with polymeric films have been extremely ${ } \mathrm{w}^{25-28}$. Comparatively, vapour-gap membranes enable relatively high thermo-osmotic fluxes and were thus the focus of this work.

For successful operation of the system, the driving force from the temperature difference must be greater than that of the opposing hydraulic pressure gradient. Increases in temperature and hydraulic pressure both result in an elevated partial vapour pressure as described by the Antoine and Kelvin equations ${ }^{29-31}$. From these relationships, it is evident that the driving force available from small temperature gradients across the membrane is equivalent to very high hydraulic pressure differences (Fig. 1b and Supplementary Note 1 ). For example, applying a $25^{\circ} \mathrm{C}$ hot temperature and a $20^{\circ} \mathrm{C}$ cold temperature is equalled by a hydraulic pressure difference of over 400 bar.

\section{Demonstration of power generation}

To generate substantial hydraulic pressures, vapour-gap membranes must be able to resist wetting of pores and maintain an open structure that facilitates transport of vapour through the membrane. Previously, hydrophobic porous membranes were not considered for pressurized applications due to the tendency of the air gap within the membrane pores to be displaced under relatively mild hydraulic pressure differences ${ }^{32}$. According to the Young-Laplace equation, the required properties for high pressure resistance are a small pore size and high hydrophobicity. Polytetrafluoroethylene (PTFE) membranes were employed with a nominal pore diameter of $20 \mathrm{~nm}$, a measured porosity of $0.77 \pm 0.02$, and an open fibrous structure (Fig. 2a). The 43- $\mu$ m-thick PTFE layer of the membrane was supported by a nonwoven polyester mesh, resulting in a total membrane thickness of $136 \mu \mathrm{m}$ (Fig. 2b). A high hydrophobicity was observed in the PTFE layer, with a measured average water contact angle of $132^{\circ}$ (Fig. 2c).

Hydraulic pressure generation across the membranes was first demonstrated in small batch experiments. In these initial tests, the partial vapour pressure difference used to drive vapour flux across the membrane originated from a solute concentration difference, rather than a temperature difference. The use of a solute concentration difference produced a more constant partial vapour pressure difference than temperature, and circumvented the need to constantly heat and cool an insulated set-up. A $5 \mathrm{M} \mathrm{NaCl}$ solution with a depressed partial vapour pressure was placed on one side of the membrane in a sealed reservoir, and deionized (DI) water was placed on the opposite side of the membrane open to the atmosphere (Fig. 2d). The vapour pressure driving force across the membrane was theoretically equivalent to a $1.9^{\circ} \mathrm{C}$ temperature difference, assuming a cold temperature of $20^{\circ} \mathrm{C}$ (Supplementary Note 2). After placing the membrane between the two reservoirs, the hydraulic pressure of the sealed reservoir was continuously monitored. Vapour transport across the membrane was confirmed by a steady increase in the hydraulic pressure of the sealed reservoir as water condensed on the low vapour pressure side of the membrane, reaching up to 13 bar after $4.7 \mathrm{~h}$ (Fig. 2e). Electrical conductivity measurements showed no salt transfer into the DI water reservoir during this period (Supplementary Fig. 1), indicating the robust air gap within the membrane pores was impervious to liquid penetration before reaching a hydraulic pressure difference of 13 bar.

Power production was examined in temperature-driven vapour flux experiments, where crossflow on either side of the membrane maintained fixed temperatures (Fig. 2f). In these experiments, the net water flux across the membrane from the hot to the cold side was monitored for various hydraulic pressure and temperature differences. At a hydraulic pressure difference of 10.3 bar, water fluxes of $5.39 \pm 0.17,7.75 \pm 0.69$ and $12.29 \pm 1.021 \mathrm{~m}^{-2} \mathrm{~h}^{-1}$ were produced for temperature differences of 20,30 and $40^{\circ} \mathrm{C}$, respectively (Supplementary Table 1). Using the water flux measurements, the power output normalized by membrane area (that is, areal power density) was determined (Supplementary Note 3 ). The highest experimental power density was $3.53 \pm 0.29 \mathrm{~W} \mathrm{~m}^{-2}$ with a $40{ }^{\circ} \mathrm{C}$ temperature difference at 10.3 bar, whereas a power output of $1.55 \pm 0.05 \mathrm{~W} \mathrm{~m}^{-2}$ was achievable with a $20^{\circ} \mathrm{C}$ temperature difference at the same pressure (Fig. 2g). Lower operating hydraulic pressures resulted in less power obtainable, with power outputs at 3.4 bar of $1.25 \pm 0.06$ and $0.50 \pm 0.08 \mathrm{~W} \mathrm{~m}^{-2}$ for 40 and $20^{\circ} \mathrm{C}$ temperature differences, respectively. The flux measurements used to determine power production represent the first implementation of hydrophobic vapour-gap membranes in a pressurized process discussed in the literature. The measured power output in the TOEC process demonstrated here is also comparable to that achieved in existing membrane-based power generation processes, such as pressureretarded osmosis and reverse electrodialysis, emphasizing the promise of this proposed system ${ }^{33-35}$.

Thermo-osmotic performance behaviour was largely in agreement with predictions from kinetic models for mass and heat transfer. Vapour transport across membranes with a pore diameter less than the mean free path of a water molecule is expected to occur primarily through Knudsen diffusion, where molecular collisions with the pore walls dominate the overall membrane transport resistance $^{36-38}$. Heat transport across the membrane also limits the vapour flux achievable. Both the latent heat associated with vaporization and conductive heat transferred across the membrane result in the formation of thermal boundary layers on either side of the membrane, in a phenomenon known as temperature polarization, which reduces the temperature difference between liquidvapour interfaces on either side of the membrane relative to the bulk 


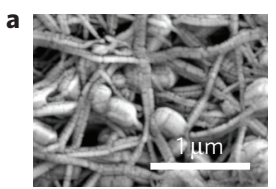

d
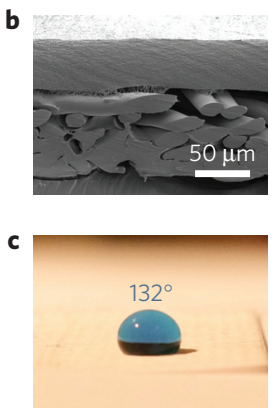
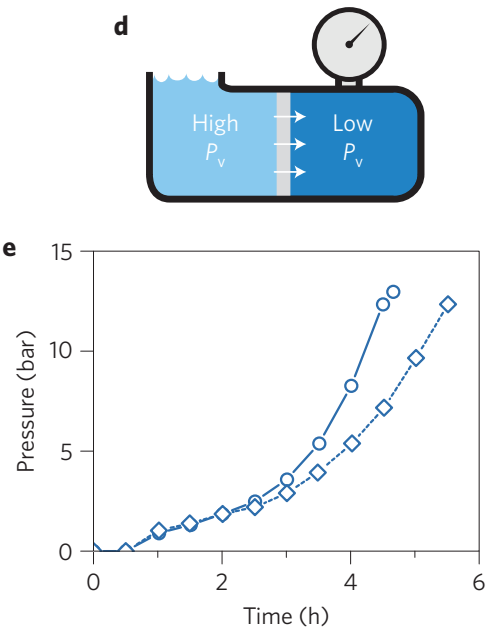

f

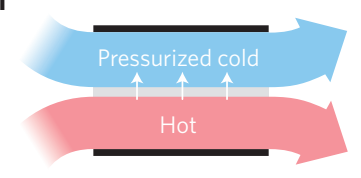

g

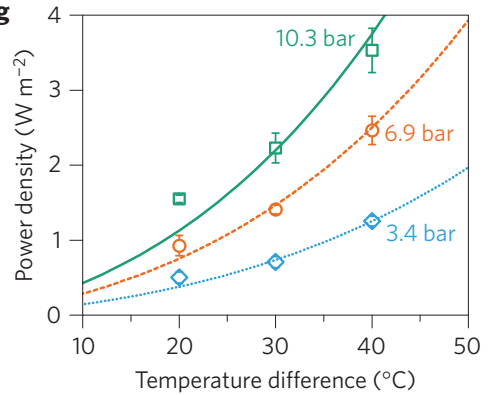

Figure 2 | Demonstration of power generation using a vapour-gap membrane. a, SEM micrograph of polytetrafluoroethylene (PTFE) membrane top surface. $\mathbf{b}$, Cross-section image of the PTFE hydrophobic layer and polyester supporting mesh. c. Average sessile contact angle of a water droplet on the membrane surface. $\mathbf{d}$, Schematic of pressure generation set-up where a membrane is placed between a low partial vapour pressure $5 \mathrm{M} \mathrm{NaCl}$ salt solution (low $P_{v}$ ) in a sealed reservoir and deionized water with a higher partial vapour pressure (high $P_{v}$ ) in an open reservoir. The partial vapour pressure difference across the membrane is theoretically equivalent to a $1.9^{\circ} \mathrm{C}$ temperature difference with a cold temperature of $20^{\circ} \mathrm{C}$. e, $\mathrm{Hydraulic}$ pressure generated in the sealed reservoir over time. Duplicate runs using separate membrane coupons are shown, with temperature maintained at $20 \pm 1{ }^{\circ} \mathrm{C}$. f, Schematic of the temperature-driven flux experiment used to measure the power output of the system. Crossflow was maintained on both sides of the membrane. $\mathbf{g}$, Experimental power density obtainable in the system as a function of the temperature difference between the bulk streams with operating pressures of $3.4 \mathrm{bar}$ (blue diamonds), $6.9 \mathrm{bar}$ (red circles) and $10.3 \mathrm{bar}$ (green squares). The cold source temperature is fixed at $20^{\circ} \mathrm{C}$. Error bars indicate the standard deviation for three experimental runs with different membrane coupons. Lines show the expected performance determined from models accounting for temperature polarization and conductive heat transfer (Supplementary Note 4).

temperature difference ${ }^{31}$. A flux prediction model that accounts for temperature polarization effects showed a strong fit to experimental measurements (lines in Fig. 2g and Supplementary Note 3) and was used to estimate the vapour permeability coefficient of the membrane to be $1731 \mathrm{~m}^{-2} \mathrm{~h}^{-1} \operatorname{bar}^{-1}\left(4.81 \times 10^{-7} \mathrm{~kg} \mathrm{~m}^{-2} \mathrm{~s}^{-1} \mathrm{~Pa}^{-1}\right)$. This experimentally determined vapour permeability coefficient was similar to that calculated from Knudsen transport models using the approximate membrane thickness, porosity, tortuosity and pore size (equal to $1501 \mathrm{~m}^{-2} \mathrm{~h}^{-1}$ bar $^{-1}$ or $4.19 \times 10^{-7} \mathrm{~kg} \mathrm{~m}^{-2} \mathrm{~s}^{-1} \mathrm{~Pa}^{-1}$ ), supporting the assumption that Knudsen transport was predominantly occurring across the membrane (Supplementary Note 4).

\section{Evaluation of temperature-driven flux}

Vapour flux and pore wetting behaviour were further evaluated using additional experiments with temperature-driven flux under crossflow (Fig. 3a,b). The net water flux across the membrane from the hot to the cold side was monitored under increasing cold reservoir hydraulic pressures (Fig. 3c). The net flux comprises a pure vapour flux from the hot to the cold side of the membrane through the air gap and a detrimental liquid water flux through wetted pores in the opposite direction driven by the increased hydraulic pressure in the cold reservoir. To distinguish between vapour flux and liquid water flux, the pressurized cold stream was dosed with salt to reach a $0.1 \mathrm{M} \mathrm{NaCl}$ concentration, and the electrical conductivity of the unpressurized hot stream was continuously monitored to detect liquid water passage carrying the non-volatile salt. The transmembrane fluxes solely attributable to vapour transport and liquid water leakage are shown separately in Figs $3 \mathrm{~d}$ and e, respectively.

Distinguishing between pure vapour flux and detrimental liquid flux across the membrane allows for a detailed evaluation of membrane performance at different pressures. For hydraulic pressures below 12 bar, electrical conductivity measurements in the hot reservoir indicated near negligible liquid water transfer across the membrane (Fig. 3e), demonstrating that the mass flux across the membrane occurred solely in the vapour phase (Fig. 3a), as expected. However, even when no pore wetting was observed, there was a slight decrease in the water flux as the hydraulic pressure in the cold reservoir increased. This decrease in flux is greater than what would be expected from the effect of hydraulic pressure on the partial vapour pressure difference across the membranedetermined to be less than $1 \%$ in this pressure range-and can be attributed to compaction of the highly porous PTFE layer, which reduces the permeability of the membrane (Supplementary Note 5). Increasing the hydraulic pressure difference beyond 12 bar resulted in a more substantial decline in the net water flux; electrical conductivity measurements indicated a majority of the flux loss was due to liquid water leakage across the membrane (Fig. 3e). However, there was also a notable decrease in the vapour flux as the pressure was increased (Fig. 3d), probably due to cooling on the hot side of the membrane as liquid water is transported directly through pores, causing increased temperature polarization (Fig. 3b). The wetting pressure of the membrane is dependent on capillary forces and described by the Young-Laplace equation ${ }^{39}$. From the measured wetting pressure, we estimate the diameter of the largest membrane pores to be $77 \mathrm{~nm}$ (Supplementary Note 6).

\section{Energy efficiency analysis}

In full-scale TOEC systems, large membrane modules will be utilized to obtain the sizable flows necessary for power generation. One possible full-scale configuration of the process is a closedloop system, which can be operated with improved efficiency by employing a heat exchanger to recover the latent heat of vaporization and conductive heat transferred across the membrane. Approximating the efficiency of this system requires accounting for various mass and heat flows. In the closed-loop configuration shown schematically in Fig. 4a, continuous operation is utilized with countercurrent flow in the membrane module and heat exchanger. The hot stream at the temperature of the heat source, $T_{H}$, enters the membrane module as the feed stream, and the pressurized cold stream at the temperature of heat sink, $T_{\mathrm{C}}$, enters the opposite end of the membrane module as the permeate stream. The feed stream 

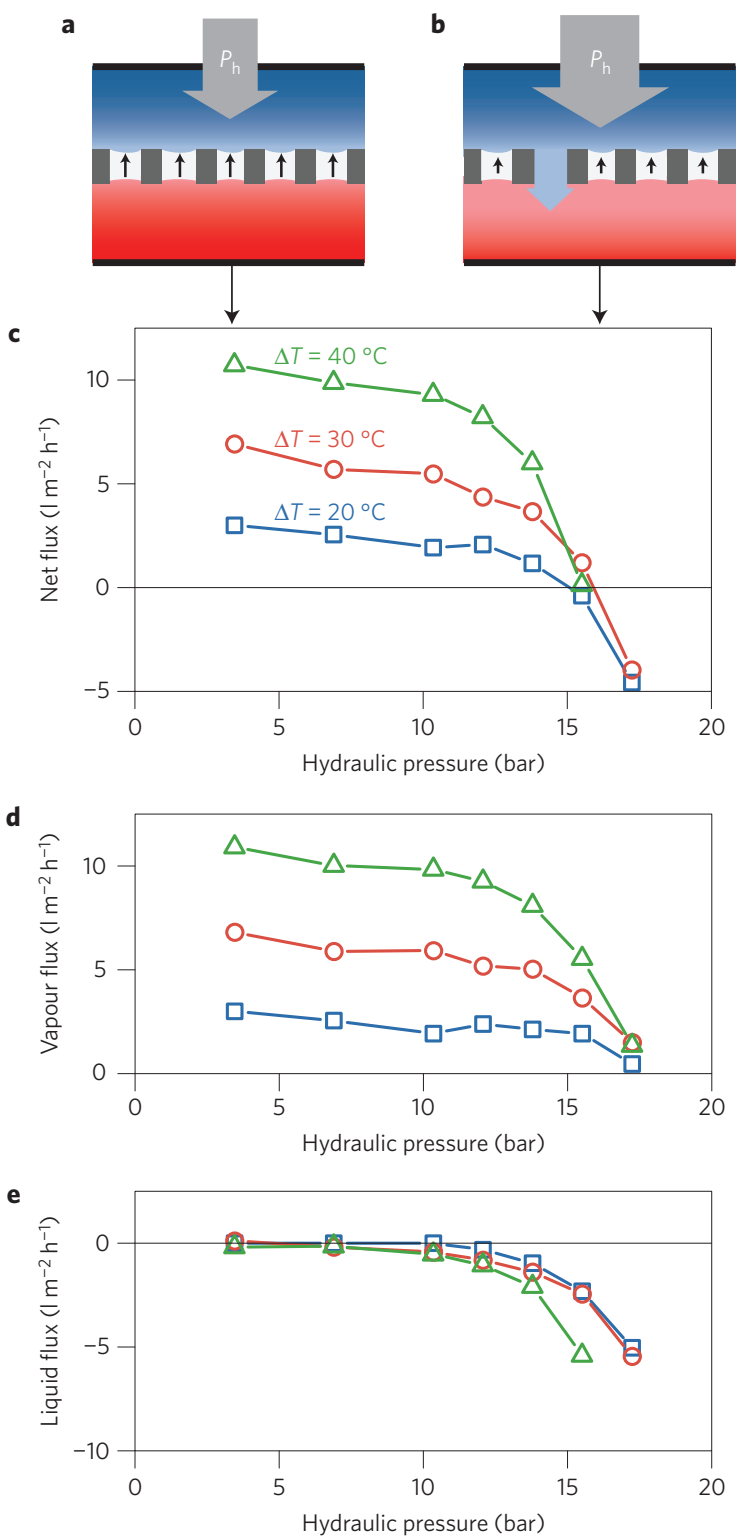

Figure $\mathbf{3}$ | Water flux across a membrane driven by a temperature gradient. a, Diagram of membrane operating at low pressure differences, where vapour is transferred from the hot feed at ambient pressure (red) to the cold permeate under hydraulic pressure, $P_{\mathrm{h}}$ (blue). b, Membrane operating under increased hydraulic pressure with some liquid water leakage from the cold to the hot side (blue arrow). Colour intensity indicates the relative temperature in either channel. c, Net transmembrane water flux as a function of the hydraulic operating pressure difference. The net flux includes both vapour and liquid water transport, where positive flux is transferred from the hot reservoir at ambient pressure to the pressurized cold reservoir. Various temperature differences across the membrane, $\Delta T$, were used with a $20^{\circ} \mathrm{C}$ cold temperature. $\mathbf{d}$, Component of the net flux across the membrane attributable solely to vapour transport from the hot to the cold side of the membrane. e, Liquid water leakage across the membrane through wetted pores from the cold pressurized side of the membrane to the hot side at ambient pressure (that is, negative flux).

transfers mass and heat across the membrane into the permeate stream, and the flow rate and temperature on both sides of the membrane module change gradually. The mass transferred into the pressurized permeate stream is directed through a turbine to generate power, while the heat transferred into the permeate stream is partially recovered in a heat exchanger.
In efficient countercurrent systems, a fixed temperature difference at any point in the membrane module can be approximated $^{40}$, which comprises two distinct components (Fig. 4b). The first component is the threshold temperature difference, $\Delta T_{\text {th }}$, describing the temperature difference that is required to overcome the partial vapour pressure difference caused by the hydraulic pressure gradient across the membrane; a system operating with a temperature difference across the membrane equal to $\Delta T_{\text {th }}$ will therefore have no net mass flux across the membrane. The second component is the excess temperature difference that drives mass flux, $\Delta T_{M}$; a system with a given $\Delta T_{M}$ value will always have the same temperature difference driving vapour and heat flux, irrespective of the hydraulic pressure used. The relationship between the transmembrane flux and the fixed temperature difference is discussed in greater detail in Supplementary Note 7.

The efficiency of the proposed closed-loop system is calculated as the work output divided by the thermal energy input. Details of the calculation and equations to determine efficiency are described in Supplementary Note 7. Efficiencies for a system with a $60^{\circ} \mathrm{C}$ heat source and a $20^{\circ} \mathrm{C}$ heat sink are shown in Fig. $4 \mathrm{c}$ as a function of the hydraulic operating pressure. We note that our analysis is extended to unrealistically high hydraulic pressures to comprehensively characterize the theoretical behaviour of the system for broad applicability to future systems. Four representative scenarios are shown with different excess temperature differences, $\Delta T_{\mathrm{M}}$, in the membrane module. In the first scenario, there is an infinitesimally small excess temperature difference between the feed and permeate streams $\left(\Delta T_{\mathrm{M}} \approx 0{ }^{\circ} \mathrm{C}\right)$. This condition occurs at the thermodynamic limit of operation, where we assume an infinitely large membrane area, and represents the upper bound of efficiency. As the hydraulic pressure approaches zero, the efficiency approaches the Carnot efficiency for a $60^{\circ} \mathrm{C}$ heat source and a $20^{\circ} \mathrm{C}$ heat sink (that is, $12 \%$ ). Realistic systems, however, will require significant excess temperature differences across the membrane module to drive vapour transport, and efficiencies are also shown with $\Delta T_{\mathrm{M}}$ values of 1,5 and $10^{\circ} \mathrm{C}$. For all of these curves, we observe a dramatic dependence of system efficiency on pressure. Within the practical range of operating pressures from 10 to 150 bar (Fig. $4 \mathrm{c}$ inset), efficiencies of $1-7 \%$ can be achieved (8-58\% of the Carnot efficiency). Minimizing the temperature difference across the modules and increasing the hydraulic pressure difference are both critical to reaching high efficiencies. Higher temperature differences between the heat source and heat sink can also increase the efficiency of the process (Fig. 4d). When source temperature differences greater than approximately $20^{\circ} \mathrm{C}$ are implemented, the efficiency of the system nearly scales with Carnot efficiency, emphasizing the effectiveness of the process with a wide range of source temperatures. Further details on the influence of heat exchanger efficiency and membrane parameters on system productivity are shown in Supplementary Note 8 and Supplementary Fig. 6. Considering the low-temperature range and the inherent limit of the Carnot efficiency, the achievable thermal efficiencies in this process are favourable compared to those of thermo-electrochemical systems, which are typically less than 1\% (refs 14-16). Furthermore, the maximum theoretical efficiency achievable is greater than that which is predicted for other membrane-based power generation technologies ${ }^{41,42}$. We note that our calculations do not account for the parasitic energy losses due to pumping working fluids through the system, which will decrease the overall efficiency.

\section{Conclusions}

In this study, a TOEC technology to harvest low-grade heat is introduced. We show that nanoporous hydrophobic membranes can effectively trap air even under hydraulic pressures up to $13 \mathrm{bar}$, and power densities of up to $3.53 \pm 0.29 \mathrm{~W} \mathrm{~m}^{-2}$ can be achieved in a 
a
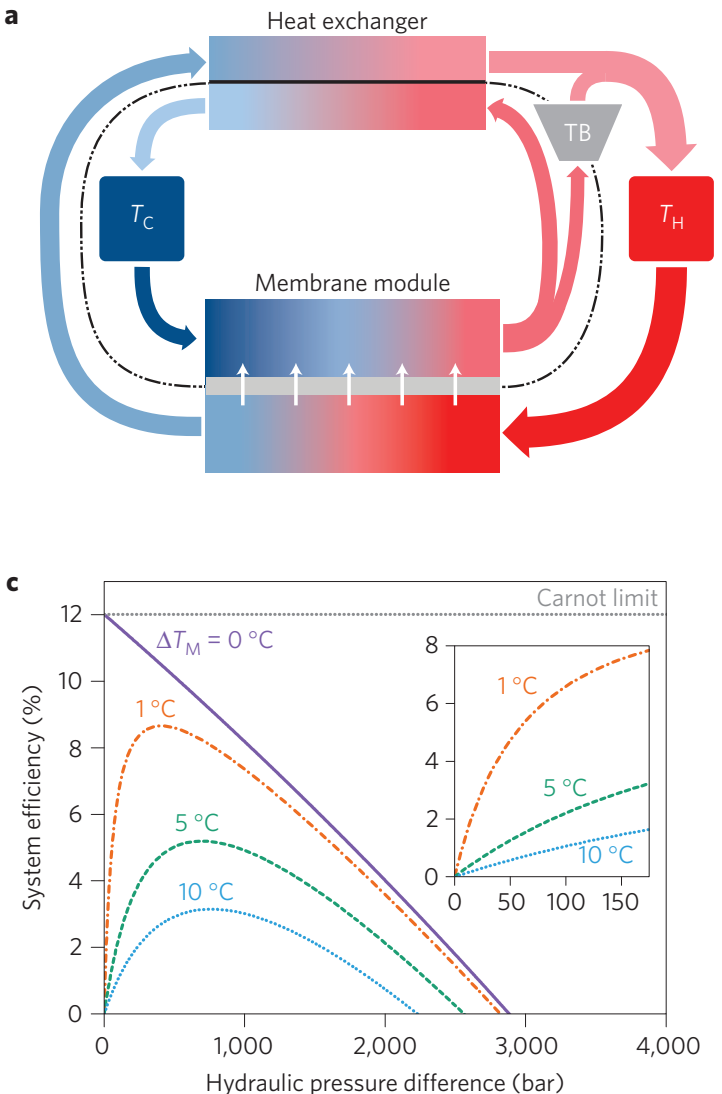

b

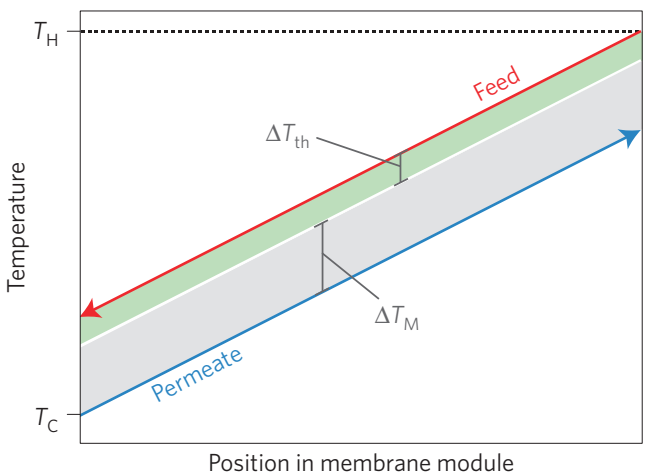

d

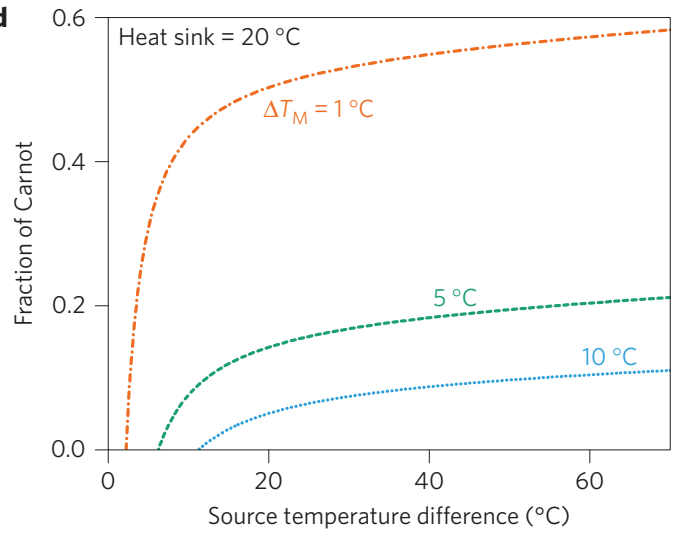

Figure 4 | Efficiency of a closed-loop system with heat recovery. a, Full-scale power generation system utilizing a continuous operation vapour-gap membrane module and a heat exchanger. The system operates with the heat source at the hot temperature, $T_{\mathrm{H}}$, and a heat sink at the cold temperature, $T_{C}$. Power is obtained by depressurizing the transmembrane flow through a turbine (TB) to produce mechanical work. The dotted line encircles the portion of the system under increased hydraulic pressure. $\mathbf{b}$, Temperature profiles in the membrane module of the hot feed stream (red arrow) and cold permeate stream (blue arrow) used in module-scale efficiency calculations. The difference between the feed and permeate temperatures comprises the fixed temperature difference that drives flux, $\Delta T_{\mathrm{M}}$, and the threshold temperature difference, $\Delta T_{\text {th }}$, that accounts for the reduction of partial vapour pressure driving force due to hydraulic pressure on the permeate side. c, Efficiency of the system with heat recovery as a function of the hydraulic pressure difference across the hydrophobic membrane. Various temperature differences at any point in the membrane module, $\Delta T_{M}$, are used. The heat source temperature is $60^{\circ} \mathrm{C}$ and the heat sink temperature is $20^{\circ} \mathrm{C}$. The inset shows efficiency results for a realistic operating pressure range below 150 bar. d, Fraction of the Carnot efficiency obtainable with increasing temperature differences between the heat source and heat sink. The hydraulic pressure difference is $100 \mathrm{bar}$, and the heat sink temperature is fixed at $20^{\circ} \mathrm{C}$. In $\mathbf{c}$ and $\mathbf{d}$, all heat is assumed to be recovered in the heat exchanger and a membrane thermal efficiency of unity is assumed.

bench-scale system operating with a $60^{\circ} \mathrm{C}$ hot temperature and a $20^{\circ} \mathrm{C}$ cold temperature. The efficiency of a continuous closed-loop system with heat recovery is projected to be greater than $50 \%$ of the Carnot efficiency with an optimized system. The proposed process offers significant advantages over existing systems since it is able to operate with heat source temperatures less than $80^{\circ} \mathrm{C}$ and has the versatility to utilize fluctuating source temperatures. Water can also be used as the sole working fluid, making it environmentally benign. To further advance the system, the development of small pore size vapour-gap membranes with improved pressure resistance will be essential. Improvements in system design may also be possible using innovative heating configurations, batch operation and alternative working fluids. With the demonstration of the process in this study and further technical advancements, the massive amount of energy available from low-grade heat sources will be obtainable using a simple, versatile system.

\section{Methods}

Membrane characterizations. A flat-sheet polytetrafluoroethylene (PTFE) membrane (Pall Corporation) with a nominal pore diameter of $20 \mathrm{~nm}$ was used in performance experiments. The membrane consisted of a PTFE layer $(43 \mu \mathrm{m}$ thick) on top of an unwoven polyester supporting mesh (total thickness of
$136 \mu \mathrm{m}$ ). Scanning electron microscopy (SEM) was used to image the membrane, and surface hydrophobicity was evaluated using an optical tensiometer (Biolin Scientific). Membrane porosity was measured gravimetrically using isopropanol to wet the membrane pores.

Pressure generation experiments. Experiments to monitor pressure generation with the PTFE membrane were conducted using a $5.1 \mathrm{~cm}^{2}$ membrane sample mounted between a sealed reservoir filled with $5 \mathrm{M} \mathrm{NaCl}$ and a reservoir with DI water open to the atmosphere. The sealed reservoir and the DI water reservoir had volumes of $15 \mathrm{ml}$ and $300 \mathrm{ml}$, respectively. A pressure gauge was used to monitor the pressure in the sealed container, and a calibrated electrical conductivity probe was placed in the DI water to measure any salt leakage across the membrane. Experiments were conducted at $20 \pm 1{ }^{\circ} \mathrm{C}$ for at least $8 \mathrm{~h}$.

Measurement of temperature-driven flux. The vapour flux across the membrane with various temperature and pressure gradients was measured in a custom-built set-up with constant crossflow on both sides of a $34 \mathrm{~cm}^{2}$ membrane coupon (Supplementary Fig. 7). Temperature was controlled by recirculating chillers and monitored using thermocouples mounted before and after the membrane cell. Pressure (0-20 bar) and crossflow velocity $\left(23.1 \mathrm{~cm} \mathrm{~s}^{-1}\right)$ on the cold side of the membrane were controlled by a high-pressure pump, back-pressure regulator and needle valve. Crossflow velocity $\left(15.6 \mathrm{~cm} \mathrm{~s}^{-1}\right)$ on the hot side was controlled by a gear pump. Mass flux across the membrane was monitored using a balance beneath the hot container. The cold pressurized water was dosed with sodium chloride before testing to reach a $0.1 \mathrm{M}$ concentration, and the conductivity of 
the water in the hot reservoir was measured to determine the liquid water leakage across the membrane. To support the membrane under pressure and facilitate crossflow, a fabric spacer was placed in the unpressurized channel between the membrane and the cell wall ${ }^{43}$. The membrane was compacted at 10.3 bar for 30 min before measurements.

Performance modelling. The projected power density of the system was calculated using existing models that account for heat transfer boundary layers on either side of the membrane and heat conduction across the membrane $e^{44-46}$, which were modified to account for the effect of hydraulic pressure on the partial vapour pressure at an interface, a relationship described by the Kelvin equation ${ }^{29}$. Experimental data were fitted to models to determine the membrane vapour permeability coefficient and used to project power densities (Supplementary Note 3). Efficiency modelling of a full-scale continuous system with heat recovery was performed using mass and heat balances; the formulation of the efficiency estimation is described in Supplementary Note 7.

\section{Received 7 February 2016; accepted 24 May 2016; published 27 June 2016}

\section{References}

1. Chu, S. \& Majumdar, A. Opportunities and challenges for a sustainable energy future. Nature 488, 294-303 (2012).

2. Chen, H., Goswami, D. Y. \& Stefanakos, E. K. A review of thermodynamic cycles and working fluids for the conversion of low-grade heat. Renew. Sustain. Energy Rev. 14, 3059-3067 (2010).

3. Gingerich, D. B. \& Mauter, M. S. Quantity, quality, and availability of waste heat from United States thermal power generation. Environ. Sci. Technol. 49, 8297-8306 (2015).

4. Hentricks, T. \& Choate, W. T. Engineering Scoping Study of Thermoelectric Generator Systems for Industrial Waste Heat Recovery (U.S. Department of Energy, 2006).

5. Blackwell, D. et al. Temperature-at-depth maps for the conterminous U.S. and geothermal resource estimates. Geotherm. Resour. Counc. Trans. 35, 1545-1550 (2011).

6. Mills, D. Advances in solar thermal electricity technology. Sol. Energy 76, 19-31 (2004).

7. Barbier, E. Geothermal energy technology and current status: an overview. Renew. Sustain. Energy Rev. 6, 3-65 (2002).

8. Tchanche, B. F., Lambrinos, G., Frangoudakis, A. \& Papadakis, G. Low-grade heat conversion into power using organic Rankine cycles-a review of various applications. Renew. Sustain. Energy Rev. 15, 3963-3979 (2011).

9. Hung, T. C., Shai, T. Y. \& Wang, S. K. A review of organic Rankine cycles (ORCs) for the recovery of low-grade waste heat. Energy 22, 661-667 (1997).

10. Vélez, F. et al. A technical, economical and market review of organic Rankine cycles for the conversion of low-grade heat for power generation. Renew. Sustain. Energy Rev. 16, 4175-4189 (2012).

11. Bell, L. E. Cooling, heating, generating power, and recovering waste heat with thermoelectric systems. Science 321, 1457-1461 (2008).

12. Zebarjadi, M., Esfarjani, K., Dresselhaus, M. S., Ren, Z. F. \& Chen, G. Perspectives on thermoelectrics: from fundamentals to device applications. Energy Environ. Sci. 5, 5147-5162 (2012).

13. Zhang, F., Liu, J., Yang, W. \& Logan, B. E. A thermally regenerative ammonia-based battery for efficient harvesting of low-grade thermal energy as electrical power. Energy Environ. Sci. 8, 343-349 (2015).

14. Zhang, F., LaBarge, N., Yang, W., Liu, J. \& Logan, B. E. Enhancing low-grade thermal energy recovery in a thermally regenerative ammonia battery using elevated temperatures. ChemSusChem 8, 1043-1048 (2015).

15. Peljo, P., Lloyd, D., Doan, N., Majaneva, M. \& Kontturi, K. Towards a thermally regenerative all-copper redox flow battery. Phys. Chem. Chem. Phys. 16, 2831-2835 (2014).

16. $\mathrm{Hu}, \mathrm{R}$. et al. Harvesting waste thermal energy using a carbon-nanotube-based thermo-electrochemical cell. Nano Lett. 10, 838-846 (2010).

17. Lee, S. W. et al. An electrochemical system for efficiently harvesting low-grade heat energy. Nature Commun. 5, 3942 (2014).

18. Abraham, T. J., MacFarlane, D. R. \& Pringle, J. M. High Seebeck coefficient redox ionic liquid electrolytes for thermal energy harvesting. Energy Environ. Sci. 6, 2639-2645 (2013).

19. Bocquet, L. Nanofluidics: bubbles as osmotic membranes. Nature Nanotech. 9 , 249-251 (2014)

20. Alkhudhiri, A., Darwish, N. \& Hilal, N. Membrane distillation: a comprehensive review. Desalination 287, 2-18 (2012).

21. Mengual, J. I., Ortiz de Zárate, J., Peña, L. \& Velázquez, A. Osmotic distillation through porous hydrophobic membranes. J. Membr. Sci. 82, 129-140 (1993).

22. Lee, J., Laoui, T. \& Karnik, R. Nanofluidic transport governed by the liquid/vapour interface. Nature Nanotech. 9, 317-323 (2014).
23. El-Bourawi, M. S., Ding, Z., Ma, R. \& Khayet, M. A framework for better understanding membrane distillation separation process. J. Membr. Sci. 285, 4-29 (2006).

24. Cath, T. Y., Adams, V. D. \& Childress, A. E. Experimental study of desalination using direct contact membrane distillation: a new approach to flux enhancement. J. Membr. Sci. 228, 5-16 (2004).

25. Dariel, M. \& Kedem, O. Thermoosmosis in semipermeable membranes. J. Phys. Chem. 79, 336-342 (1975).

26. Mengual, J. I. \& Aguilar, J. Thermoosmosis of water through cellulose acetate membranes. J. Membr. Sci. 4, 209-219 (1978).

27. Tasaka, M., Mizuta, T. \& Sekiguchi, O. Mass transfer through polymer membranes due to a temperature gradient. J. Membr. Sci. 54, 191-204 (1990).

28. Kim, S. \& Mench, M. M. Investigation of temperature-driven water transport in polymer electrolyte fuel cell: thermo-osmosis in membranes. J. Membr. Sci. 328, 113-120 (2009)

29. Adamson, A. W. \& Gast, A. P. Physical Chemistry of Surfaces (John Wiley, 1997).

30. Lee, J. \& Karnik, R. Desalination of water by vapor-phase transport through hydrophobic nanopores. J. Appl. Phys. 108, 044315 (2010).

31. Schofield, R. W., Fane, A. G. \& Fell, C. J. D. Heat and mass transfer in membrane distillation. J. Membr. Sci. 33, 299-313 (1987)

32. Khayet, M. Membranes and theoretical modeling of membrane distillation: a review. Adv. Colloid Interface Sci. 164, 56-88 (2011)

33. Cusick, R. D., Kim, Y. \& Logan, B. E. Energy capture from thermolytic solutions in microbial reverse-electrodialysis cells. Science 335, 1474-1477 (2012).

34. Ramon, G. Z., Feinberg, B. J. \& Hoek, E. M. V. Membrane-based production of salinity-gradient power. Energy Environ. Sci. 4, 4423-4434 (2011)

35. Helfer, F., Lemckert, C. \& Anissimov, Y. G. Osmotic power with pressure retarded osmosis: theory, performance and trends-a review. J. Membr. Sci. 453, 337-358 (2014)

36. Carman, P. C. Flow of Gases Through Porous Media (Academic, 1956).

37. Kast, W. \& Hohenthanner, C. R. Mass transfer within the gas-phase of porous media. Int. J. Heat Mass Transfer 43, 807-823 (2000).

38. Matsuura, T. Synthetic Membranes and Membrane Separation Processes (CRC Press, 1994)

39. García-Payo, M., Izquierdo-Gil, M. \& Fernández-Pineda, C. Wetting study of hydrophobic membranes via liquid entry pressure measurements with aqueous alcohol solutions. J. Colloid Interface Sci. 230, 420-431 (2000).

40. Lin, S., Yip, N. Y. \& Elimelech, M. Direct contact membrane distillation with heat recovery: thermodynamic insights from module scale modeling. J. Membr. Sci. 453, 498-515 (2014).

41. Lin, S., Yip, N. Y., Cath, T. Y., Osuji, C. O. \& Elimelech, M. Hybrid pressure retarded osmosis-membrane distillation system for power generation from low-grade heat: thermodynamic analysis and energy efficiency. Environ. Sci. Technol. 48, 5306-5313 (2014).

42. McGinnis, R. L., McCutcheon, J. R. \& Elimelech, M. A novel ammonia-carbon dioxide osmotic heat engine for power generation. J. Membr. Sci. 305, 13-19 (2007)

43. Straub, A. P., Osuji, C. O., Cath, T. Y. \& Elimelech, M. Selectivity and mass transfer limitations in pressure-retarded osmosis at high concentrations and increased operating pressures. Environ. Sci. Technol. 49, 12551-12559 (2015).

44. Qtaishat, M., Matsuura, T., Kruczek, B. \& Khayet, A. Heat and mass transfer analysis in direct contact membrane distillation. Desalination 219 , 272-292 (2008)

45. Phattaranawik, J., Jiraratananon, R. \& Fane, A. G. Heat transport and membrane distillation coefficients in direct contact membrane distillation. J. Membr. Sci. 212, 177-193 (2003)

46. Khayet, M. \& Matsuura, T. Preparation and characterization of polyvinylidene fluoride membranes for membrane distillation. Ind. Eng. Chem. Res. 40, 5710-5718 (2001)

\section{Acknowledgements}

We acknowledge the National Science Foundation Graduate Research Fellowship DGE-1122492 awarded to A.P.S.

\section{Author contributions}

A.P.S., N.Y.Y., S.L., J.L. and M.E. participated in developing the process and designing the experiments. A.P.S. performed the experiments and analysed the data. A.P.S. and S.L. conducted the system modelling. A.P.S. and M.E. co-wrote the paper. All authors contributed to data analysis, discussed the results and commented on the manuscript.

\section{Additional information}

Supplementary information is available online. Reprints and permissions information is available online at www.nature.com/reprints. Correspondence and requests for materials should be addressed to M.E.

\section{Competing interests}

The authors declare no competing financial interests. 